\title{
Comportamiento de orden fraccionario en la respuesta de un circuito RC mediante derivada de núcleo singular
}

\author{
Ernesto Zambrano-Serrano, Miguel A. Platas-Garza, \\ Alejandro E. Loya-Cabrera, Guadalupe E. Cedillo-Garza, \\ Cornelio Posadas-Castillo \\ Universidad Autónoma de Nuevo León, Facultad de Ingeniería Mecánica y \\ Eléctrica, Departamento de Electrónica y Automatización. \\ cornelio.posadascs@uanl.edu.mx
}

\section{RESUMEN}

En este artículo, se presenta la ecuación diferencial fraccionaria de un circuito electrónico $R C$ en términos de la derivada fraccionaria de tipo Caputo y la solución analitica exacta usando propiedades de la transformada de Laplace y la función Mittag-Leffler. El orden de la derivada fraccionaria es definido en el intervalo $0<q \leq 1$, preservando la dimensionalidad de los parámetros $R$ y $C$. Además, se muestra la respuesta experimental del circuito propuesto y se compara con las soluciones analíticas. Los resultados muestran que el voltaje del capacitor depende directamente de los valores del orden fraccionario.

\section{PALABRAS CLAVE}

Circuito electrónico RC, cálculo fraccionario; solución analítica, derivada tipo Caputo.

\section{ABSTRACT \\ This paper proposes the fractional-order differential equation of an $R C$ electronic circuitin terms of the Caputo-type fractionalderivative. The fractional- order derivative is defined in the interval $0<q \leq 1$ considering the dimensionality of the parameters $R$ and $C$. The exact analytical solution is presented using properties of the Laplace transform and Mittag-Leffler function. Besides, the experimental response of the proposed circuit is presented and compared with the analytical solutions. The results show that the voltage depends on the values of the fractional order.}

\section{KEYWORDS}

$\mathrm{RC}$ electronic circuit, fractional calculus, analytical solution; Caputo derivative

\section{INTRODUCCIÓN}

El cálculo de orden fraccionario se ocupa del estudio de las derivadas e integrales de orden arbitrario sobre dominios reales o complejos y sus aplicaciones. Tiene sus raíces en 1695, en una carta de L'Hopital a Leibniz, donde el primero preguntó sobre el posible significado de una derivada $d^{n} / d t^{n}$ cuando $n=1 / 2$. 
En respuesta a la carta recibida, Leibniz contestó: "Es una aparente paradoja, de la cual algún día se extraerán consecuencias muy útiles". Esta respuesta atrajo la atención de brillantes científicos durante los siglos XVIII y XIX. ${ }^{1}$ De entre los cuales podemos mencionar a Euler (1738), Laplace (1812), Fourier (1822), Abel (1823), Liouville (1832-1855), Grünwald (1867), Letnikov (1868-1872), Riemann (1876), Laurent (1884) o Heaviside (1893-1912).

Recientemente, el cálculo de orden fraccionario ha experimentado avances considerables en diferentes áreas del conocimiento como física, biología, química, matemáticas e ingeniería, considerando la aplicación del cálculo fraccionario en problemas del mundo real como se muestran Su et al. ${ }^{2}$ y Tavazoei. ${ }^{3}$ Hoy en día, muchos matemáticos e ingenieros están interesados en este nuevo campo. Intentando dar respuesta a las siguientes preguntas de investigación: ¿Cómo caracterizar la derivada fraccionaria utilizada en este nuevo campo? ¿Cuál es la importancia de una derivada fraccionaria? ¿Cuándo y dónde podemos usar la derivada fraccionaria?

Las derivadas fraccionarias son operadores no locales debido a que se definen por medio de integrales. Por tanto, la derivada fraccionaria contiene información sobre la función en todos sus puntos anteriores, por lo que posee un efecto de memoria; esto significa que el siguiente estado de un sistema depende no sólo de su estado actual, sino también de todos los estados históricos. Esto es más realista y los resultados derivados de los sistemas fraccionarios son de naturaleza más general y son esenciales para una descripción y comprensión más precisa del comportamiento del sistema complejo y dinámico. ${ }^{4}$ Asimismo, las derivadas e integrales de orden fraccionario consideran propiedades hereditarias, fenómenos de relajación, fractalidad temporal a diferentes escalas, heterogeneidades materiales $\mathrm{y}$ efectos distribuidos no locales, los cuales son fundamentales para describir los componentes eléctricos. ${ }^{2}$ Otra característica del cálculo fraccionario es la inclusión de nuevos grados de libertad al sistema al incrementar la información que se puede obtener de la naturaleza del fenómeno en cuestión.

Actualmente, existen muchos tipos de derivadas fraccionarias en el cálculo fraccionario. Las definiciones más utilizadas son las propuestas por RiemannLiouville y Caputo, las cuales son definidas por medio de la convolución de la derivada local de una función dada con una función de ley de potencias, ${ }^{4}$ también conocida como derivada fraccionaria de núcleo singular. Mientras que Caputo y Fabrizio, hicieron una interesante propuesta de derivada de orden fraccionario, proponiendo un operador con núcleo local y no singular que involucra la ley de decaimiento exponencial en lugar de la ley de potencias. ${ }^{5}$ Con base en la función de Mittag-Leffler extendida generalizada no singular y no local, Atangana y Baleanu propusieron dos derivadas fraccionarias en el sentido de LiouvilleCaputo y Riemann-Liouville. Las derivadas de orden fraccionario propuestas por Atangana y Baleanu son al mismo tiempo filtros y operadores fraccionarios. ${ }^{6}$ Khalil et al. propusieron extender las definiciones de límites ordinarios de las derivadas en una función llamada derivada conformable. ${ }^{7}$

Recientemente, muchos investigadores han intentado modelar procesos reales utilizando el cálculo fraccionario. Munoz-Pacheco, et al. ${ }^{8}$ quienes estudiaron el efecto que agrega la memoria en un sistema regulador de glucosa-insulina mediante la aplicación de un operador fraccionario. Rosales et. al. ${ }^{9}$ investigaron 
la solución de un circuito RC usando diferentes tipos de operadores fraccionarios. La solución analítica del circuito RLC considerando los operadores FabrizioCaputo, Atangana-Baleanu-Caputo, Atangana-Koca-Caputo es presentada por Gómez-Aguilar. ${ }^{10}$. Ndolane Sene analiza la estabilidad asintótica local y la estabilidad asintótica global de un circuito RLC considerando el operador de Liouville-Caputo. ${ }^{11}$ También muestra la estabilidad de entrada fraccionaria, estudiando la estabilidad asintótica global uniforme de las ecuaciones diferenciales fraccionarias de los circuitos RL, RC y LC. ${ }^{12}$ De acuerdo con las referencias previamente mostradas, únicamente se han reportado soluciones analíticas y análisis de estabilidad de circuitos electrónicos en configuraciones RC, RLC, LC y RL considerando operadores de orden fraccionario sin mostrar a manera de comparación la respuesta experimental de un circuito RC. El único reporte en la literatura relacionado a la implementación de un circuito $\mathrm{RC}$ considerado operadores fraccionarios es el de Gómez-Aguilar et al. ${ }^{13}$ donde presenta el resultado obtenido en un espectroscopio aplicado a un RC.

Teniendo en cuenta tal falta, este artículo hace una contribución al tema presentando la comparación entre la implementación experimental del circuito $\mathrm{RC}$ y la solución analítica de orden fraccionario basado en la derivada fraccionaria de tipo Caputo.

El resto de artículo está organizado de la siguiente manera, primero se presentan algunos conceptos preliminares relacionados al cálculo fraccionario. Luego se muestra la solución analítica de un circuito $\mathrm{RC}$ en serie de orden fraccionario. $\mathrm{La}$ implementación experimental del circuito RC y la comparación con la respuesta de orden fraccionario se reportan después, finalmente, se presentan una serie de comentarios a modo de conclusión.

\section{DEFINICIONES DE ORDEN FRACCIONARIO}

En esta sección recordamos las principales definiciones de los operadores de orden fraccionario. La definición de la derivada fraccionaria de tipo Caputo esta expresada de la siguiente forma:

Definición $1 .{ }^{4}$ Considere una función $\mathrm{f}:[0,+\infty) \rightarrow \mathrm{R}$, la derivada fraccionaria en el sentido de Caputo de la función $f$ de orden $q$ es formulada mediante la expresión.

Para todo $t>0$, donde q es el orden de la derivada fraccionaria y $\Gamma(\cdot)$ es la función Gamma.

$$
{ }^{c} D_{t}^{q} f(t)=\frac{1}{\Gamma(1-q)} \int_{0}^{t} \frac{f^{\prime}(s)}{(t-s)^{q}} d s ; \quad n-1<q \leq n,
$$

La definición de la derivada de orden fraccionario en el sentido de Caputo es ampliamente considerada en el estudio de sistemas en el dominio del tiempo, debido a que, las condiciones iniciales para la derivada fraccionaria de tipo Caputo pueden ser expresadas de la misma forma que una ecuación diferencial ordinaria y con una interpretación física conocida. Además, de que la derivada de una constante en el sentido de Caputo es cero. 

forma

La transformada de Laplace de la ecuación (1) se define de la siguiente

$$
\mathcal{L}\left[{ }^{C} D_{t}^{q} f(t)\right]=s^{q} F(s)-\sum_{k=0}^{n-1} s^{q-k-1} f^{(k)}(0) ; \quad n-1<q \leq n,
$$

donde $f^{(k)}$ es la $k$-ésima derivada ordinaria. La transformada inversa de Laplace requiere la introducción de la función Mittag-Leffler, la cual es definida como:

Definición $2 .{ }^{4}$ La función Mittag-Leffler con dos parámetros es expresada de la siguiente forma

$$
E_{q, \beta}(z)=\sum_{k=0}^{\infty} \frac{z^{k}}{\Gamma(q k+\beta)},
$$

donde $q>0, \beta \in R, y z \in C$.

En particular cuando $q=1$ y $\beta=1$ de la ecuación (3) se tiene que

$$
E_{1,1}(z)=\sum_{k=0}^{\infty} \frac{z^{k}}{\Gamma(k+1)}=\sum_{k=0}^{\infty} \frac{z^{k}}{k !}=e^{z} .
$$

Por lo que se considera a la función exponencial como un caso particular de la función Mittag-Leffler.

\section{SOLUCIÓN DEL CIRCUITO RC CON OPERADORES LOCALES Y NO LOCALES}

En este artículo se considera el circuito eléctrico RC descrito por la figura 1.

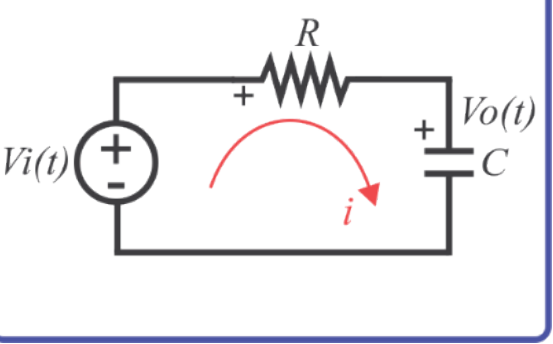

Fig.1. Circuito RC.

Considerando las leyes de voltaje de Kirchhoff, la ecuación diferencial que describe el comportamiento del circuito RC esta expresada como

$$
\frac{d V o(t)}{d t}+\frac{V o(t)}{R C}=\frac{V i(t)}{R C},
$$

donde $R C=\tau$ es la constante de tiempo del circuito, $R$ es la resistencia expresada en Ohms, C es la capacitancia medida en Faradios, $V o(t)$ es el voltaje en el capacitor, y $V i(t)$ es el voltaje de entrada. Considerando que el voltaje inicial sobre el capacitor es $V o(0)=0$, y asumiendo que la fuente $V i(t)$ corresponde a una entrada constante $V i$ la solución de la ecuación (5) en el dominio de tiempo es 


$$
V o(t)=V i\left(1-e^{-\frac{t}{\tau}}\right)
$$

la ecuación (6) describe la carga del circuito RC considerando una fuente constante. Por otra parte, preservar la dimensionalidad física al momento de considerar un operador de orden fraccionario es crucial en la aplicación. En matemáticas puras por lo general, la derivada de orden entero es remplazada por definiciones de orden fraccionario, pero, esto no es suficiente para problemas físicos y algunas aplicaciones en ingeniería. Por tanto, se requiere de una modificación dimensional para el caso fraccionario. Para este propósito, Rosales et al. ${ }^{9}$ y Gomez-Aguilar ${ }^{10}$ proponen una metodología sistemática capaz de diseñar ecuaciones diferenciales fraccionarias para sistemas físicos, la cual consiste en transformar la derivada ordinaria en una derivada fraccionaria de la siguiente forma

$$
\frac{d}{d t} \rightarrow \frac{1}{\sigma^{1-q}} \frac{d^{q}}{d t^{q}} \quad ; \quad 0<q \leq 1 \text {. }
$$

La introducción del parámetro $\sigma$ permite la consistencia dimensional cuando $\sigma=\operatorname{seg}$. Además, cuando $q=1$ la ecuación (7) es una ecuación diferencial ordinaria de primer orden con respecto al tiempo $t$.

Sustituyendo (7) en (5) obtenemos la ecuación diferencial de orden fraccionario correspondiente al circuito RC expresada de la siguiente forma

$\frac{d^{q} V o(t)}{d t^{q}}+\frac{V o(t)}{\tau^{q}}=\frac{V i(t)}{\tau^{q}} ; \quad 0<q \leq 1$,

donde $\sigma=\tau=R C$ es la constante de tiempo expresada en segundos y el operador $d^{q} / d t^{q}={ }^{C} D_{t}^{q}$ expresando en (1). Entonces la solución analítica de la ecuación diferencial fraccionaria definida por (8) se obtiene aplicando la transformada de Laplace expresada en (2) en ambos lados de la ecuación (8) y, asumiendo que la fuente $V i(t)$ corresponde a una entrada constante $V i$ obtenido la siguiente relación

$$
\begin{gathered}
s^{q} \mathcal{L}(V o(t))+\frac{1}{\tau^{q}} \mathcal{L}(V o(t))=\frac{1}{\tau^{q}} \mathcal{L}(V i), \\
V o(s)\left(s^{q}+\frac{1}{\tau^{q}}\right)=\frac{V i}{s \tau^{q}}, \\
V o(s)=\frac{V i}{s\left(s^{q} \tau^{q}+1\right)},
\end{gathered}
$$

donde $V o(s)$ es la transformada de Laplace de $V o(t)$. Considerando el método propuesto por Kenneth y $\operatorname{Ross}^{14}$ y aplicando la transformada inversa de Laplace es posible expresar (9) como una serie

$$
V o(t)=V i\left[1-\sum_{k=0}^{\infty} \frac{\left(-\frac{t}{\tau}\right)^{k q}}{\Gamma(k q+1)}\right]
$$

entonces la solución analítica de la ecuación diferencial fraccionaria (10) es expresada como: 


$$
V o(t)=V i\left[1-E_{q}\left(-\left(\frac{t}{\tau}\right)^{q}\right)\right],
$$

donde $E_{q}$ (.) representa la función Mittag-Leffler (3) $\operatorname{con} \beta=1$.

La figura 2, muestra el comportamiento en el tiempo del voltaje en el capacitor $V o(t)$ expresado en (11) considerando diferentes valores de orden fraccionario $q$. con incrementos $\Delta q=0.1$, una fuente de voltaje de entrada constante $\mathrm{Vi}=10 \mathrm{Volts}$, un Capacitor $C=1 \mu \mathrm{F}$, una Resistencia $\mathrm{R}=1 \mathrm{M} \Omega$ y $\tau=1 \mathrm{seg}$, donde se observa que conforme $q \rightarrow 0$ el voltaje de salida decrece similarmente.

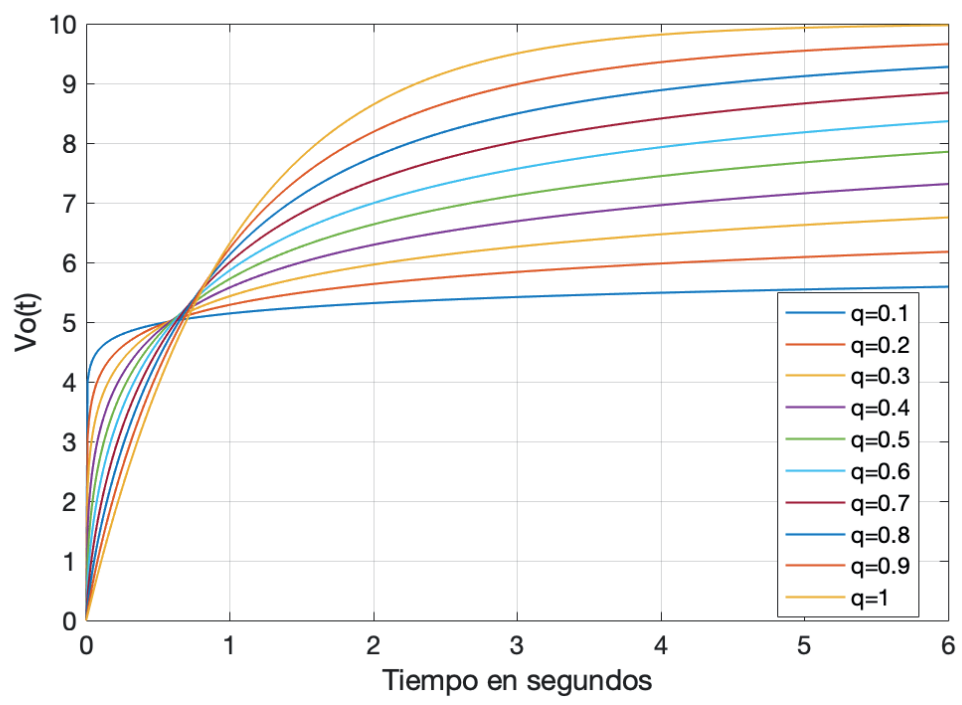

Fig. 2. Solución analítica de la ecuación (11) considerando diferentes valores de q correspondiente al voltaje en el capacitor $\mathrm{Vo}(t)$.

\section{DISEÑO EXPERIMENTAL DEL CIRCUITO RC}

Para propósitos experimentales, consideramos la plataforma NI-myRIO-1900. NI-myRIO es un dispositivo embebido el cual cuenta con entradas analógicas, salidas analógicas, además de líneas de E/S digitales, LEDs, acelerómetro un FPGA Xilinx y un procesador ARM Cortex-A9. La relativa simplicidad que ofrece el dispositivo al momento de configurarlo ha permitido su consideración en diferentes áreas tales como procesamiento de señales, sistemas control, robótica y mecatrónica.

El circuito RC mostrado en la figura 1 fue implementado de forma práctica como se muestra en la figura 3. Las principales características del dispositivo myRIO son:

Procesador Xilinx Z-7010.

Velocidad $667 \mathrm{Mhz}$.

Memoria DDR3 de 256Mb.

Salidas analógicas de \pm 10 Volts.

Resolución de 12 bits. 


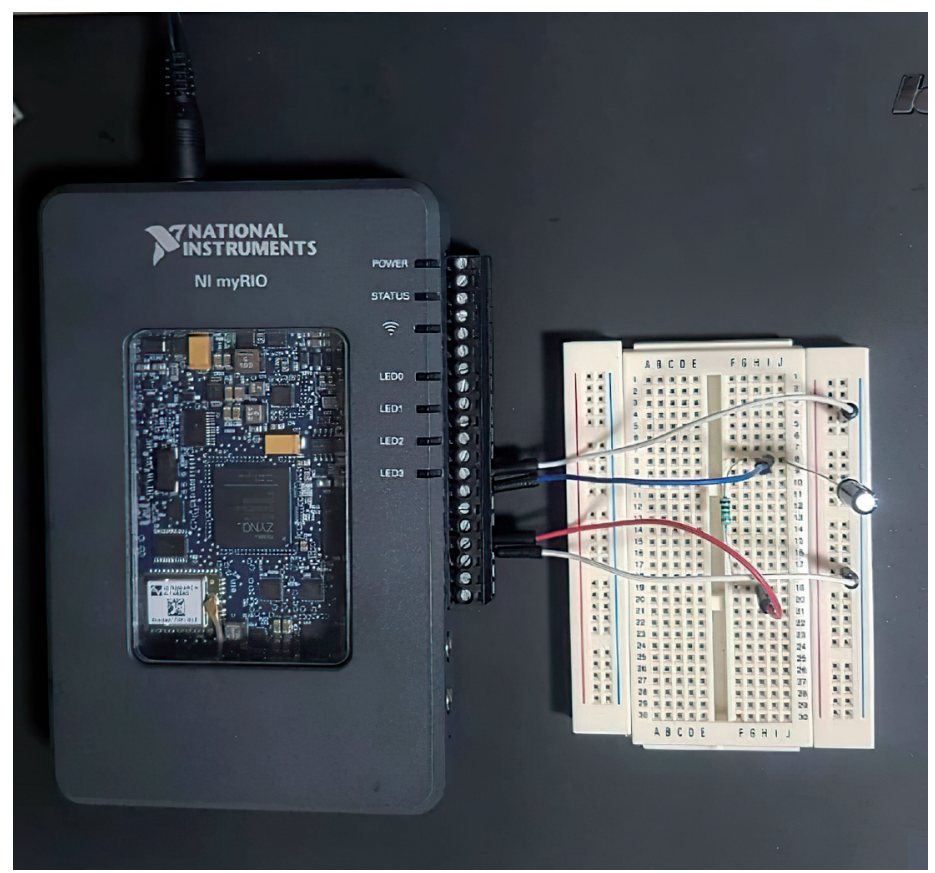

Fig. 3. Implementación electrónica del circuito RC.

El valor de los elementos pasivos es

Resistencia $\mathrm{R}=1 \mathrm{M} \Omega$.

Capacitor $\mathrm{C}=1 \mu \mathrm{F}$.

El proceso de adquisición de datos fue implementado en LabView con el objetivo de obtener los datos experimentales de la respuesta en el tiempo del circuito RC. El diseño del instrumento virtual realizado en LabView se muestra en la figura 4. La figura 4 (arriba), muestra el diseño del instrumento virtual. De la figura 4 (abajo) el bloque "analog input" es utilizado para adquirir una muestra de voltaje en el capacitor el cual se encuentra conectado al puerto de entrada analógica, mientras que el bloque "analog output" envía la señal de excitación al circuito RC mediante una salida analógica. El proceso en lazo cerrado está configurado para realizarse cada 5 milisegundos.

Además, los datos obtenidos experimentalmente son procesados en Matlab 2016b. La medición adquirida experimentalmente es comparada con la solución analítica (11) considerando la suma de los errores cuadráticos para diferentes valores de orden fraccionario $q$; la suma de los errores cuadráticos son considerados como criterio de ajuste o función de costo, la cual se realiza considerando la siguiente ecuación

$$
J=\sum_{k=1}^{N}\left(V_{o}[k]-y_{m}[k]\right)^{2}
$$

donde $V_{0}[k]$ representa la $k$-ésima muestra de la solución analítica, $y_{m}[k]$ es la señal adquirida experimentalmente y $N$ es número de datos obtenidos experimentalmente. 

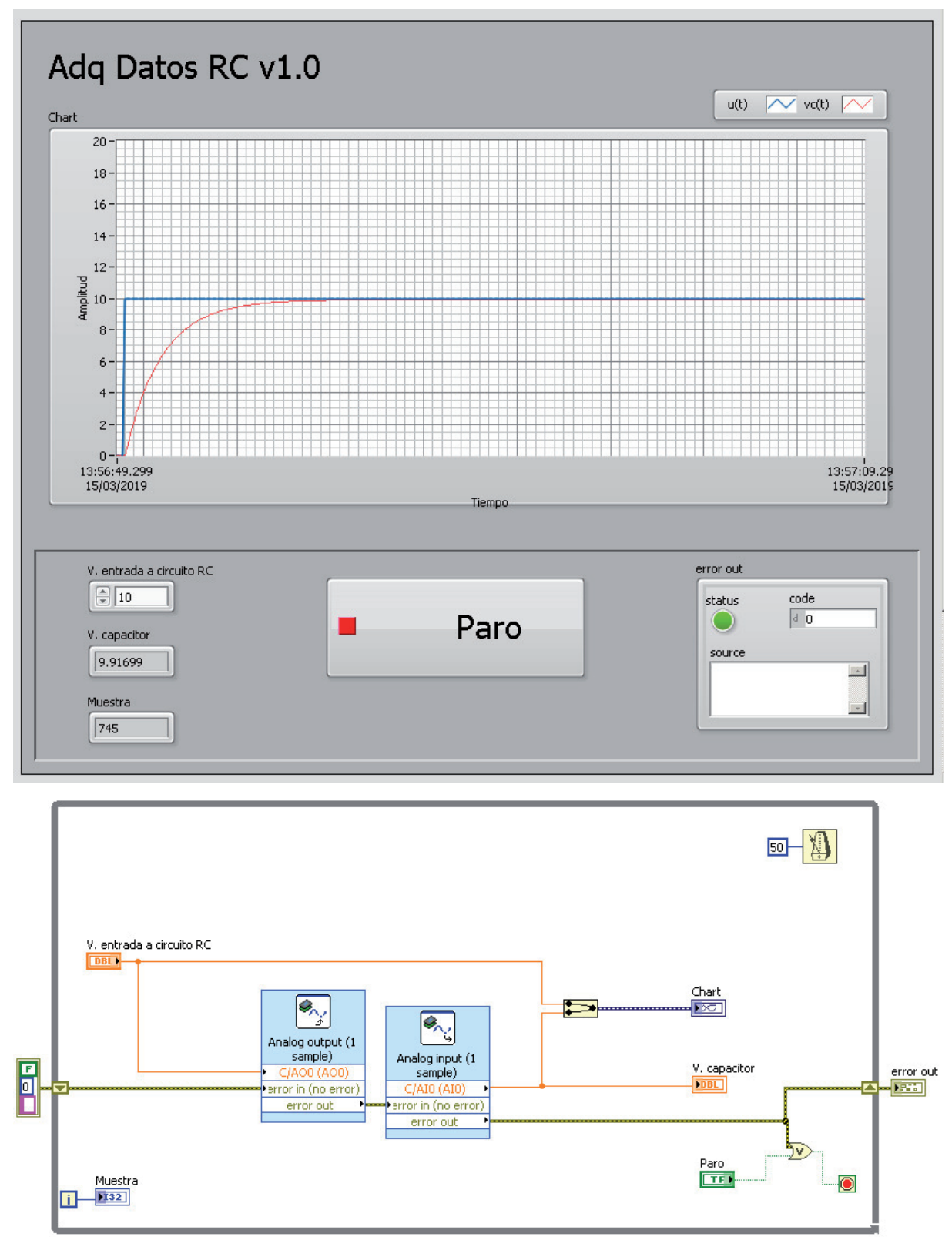

Fig. 4. Diseño del instrumento virtual en LabView. (arriba) vista frontal del instrumento, (abajo) diseño del instrumento.

La figura 5 muestra el valor de la función de costo (12) para diferentes valores de $q$, en este artículo tomamos en cuenta 80 diferentes valores de orden $q$ considerando el siguiente intervalo $q \epsilon(0.95,0.99)$. Bajo estas condiciones, se obtiene que el valor mínimo de $q$ que mejor se aproxima a la solución experimental del circuito $R C$ mostrado en la figura 1 con una resistencia $R=1 \mathrm{M} \Omega$, un capacitor $\mathrm{C}=1 \mu \mathrm{F}$ y una entrada constante de $\mathrm{Vi}=10$ volts. Bajo estas condiciones, se obtiene que el valor mínimo de $q=0.98475$.

En la figura 6 (Respuesta del circuito RC) se observa la respuesta experimental adquirida en color rojo, la solución analítica (11) considerando un orden fraccionario $q=0.98475$ en color azul y la solución analítica de orden 


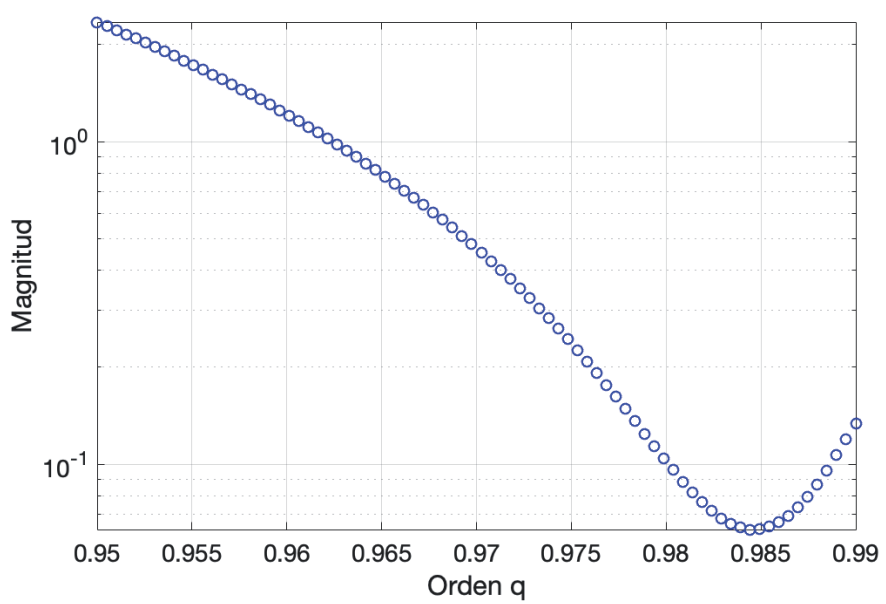

Fig. 5. Evaluación de función de costo para determinar la mejor aproximación.
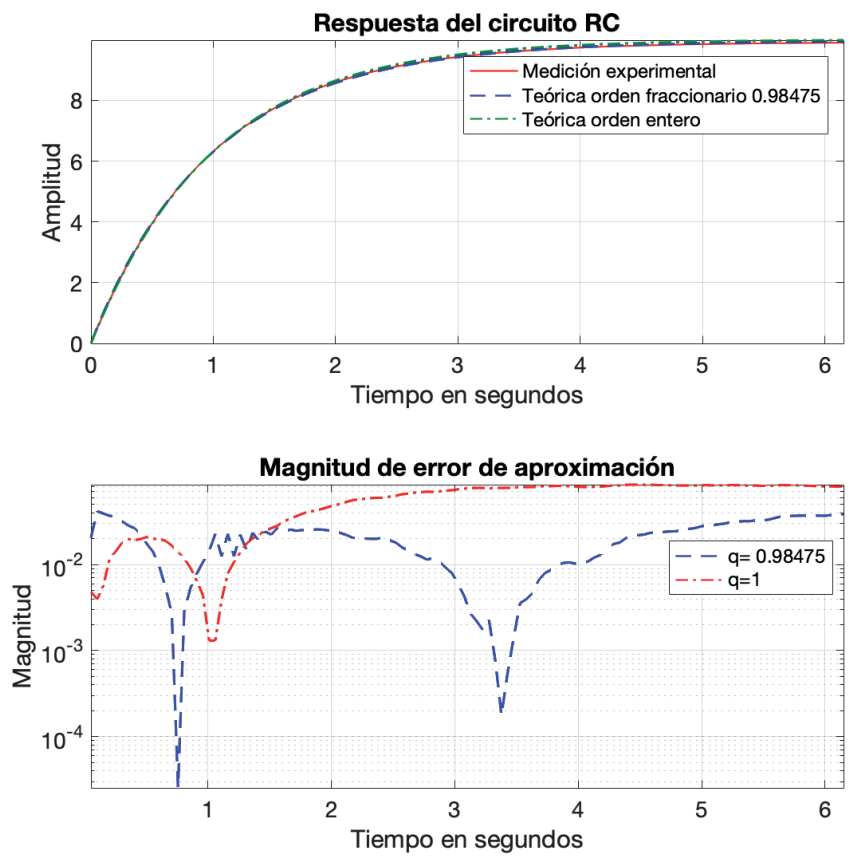

Fig. 6. Error de aproximación para la respuesta de orden fraccionario propuesta y la respuesta de orden entero.

entero mostrada en (6) en color verde. Mientras que en la figura 6 (Magnitud de error de aproximación) se muestra la magnitud de los errores de ambas aproximaciones. Note que la aproximación fraccionaria tiene un error menor que la de orden entero.

\section{COMENTARIOS FINALES}

En este artículo se estudió la solución analítica y la respuesta experimental de un circuito RC considerando la definición de la derivada fraccionaria de 
tipo Caputo, además, se consideró la metodología de diseño de ecuaciones diferenciales fraccionarias de sistemas físicos preservando la dimensionalidad de los elementos pasivos R y C. Se presentó la solución analítica considerando la transformada de Laplace y la función Mittag-Leffler para un intervalo $0<q \leq 1$. El circuito RC analizado fue implementado electrónicamente considerando la plataforma NI-myRIO-1900. Donde los resultados experimentales fueron procesados en Matlab y comparados con la solución analítica considerando diferentes valores de orden fraccionario $q$. Por medio de una función de costo se determinó el valor mínimo de orden $q$ que mejor se aproxima a los datos experimentales obtenidos por la tarjeta NI-myRIO-1900, obteniendo que la mejor aproximación ocurre cuando se considera el siguiente valor $q=0.98475$. Finalmente, se observa que conforme $q \rightarrow 0$ el voltaje de salida $V_{0}(t)$ decrece similarmente.

\section{AGRADECIMIENTOS}

E. Zambrano-Serrano agradece a CONACYT/MÉXICO (350385), y al Cuerpo Académico Automatización y Control de la UANL. C. Posadas-Castillo agradece a CONACYT/MÉXICO No. 166654, A1-S-31628. Todos los autores agradecen a la Facultad de Ingeniería Mecánica y Eléctrica de la UANL.

\section{REFERENCIAS}

1. D. Baleanu, K. Diethelm, E. Scalas and J. Trujillo, Fractional Calculus: Models and Numerical Methods, London: World Scientific Publishing, 2017.

2. H. Su, Y. Zhang, D. Baleanu, W. Chen and Y. Chen, "A new collection of real world applications of fractional calculus in science and engineering," Communications in Nonlinear Science and Numerical Simulation, vol. 64, pp. 213-231, 2018.

3. M. Tavazoei, "Fractional order chaotic systems: history, achievements, applications, and future challenges," The European Physical Journal Special Topics, vol. 229, pp. 887-904, 2020.

4. I. Podlubny, Fractional Differential Equations, New York: Academic Press, 1998.

5. M. Caputo and M. Fabrizio, "A new Definition of Fractional Derivative without singular kernel," Progress in Fractional Differentiation and Applications, vol. 1, no. 2, pp. 73-85, 2015.

6. A. Atangana and D. Baleanu, "New fractional derivatives with non-local and non-singular kernel," Thermal Science, vol. 20, no. 2, pp. 763-769, 2016.

7. R. Khalil, M. Al Horani, A. Yousef and M. Sababheh, "A new definition of fractional derivative," Journal of Computational and Applied Mathematics, vol. 264, pp. 65-70, 2014.

8. J. Munoz-Pacheco, C. Posadas-Castillo and E. Zambrano-Serrano, "The Effect of a Non-Local Fractional Operator in an Asymmetrical GlucoseInsulin Regulatory System: Analysis, Synchronization and Electronic Implementation," Symmetry, vol. 12, no. 9, p. 1395, 2020.

9. J. Rosales, J. Filoteo and G. A, "A comparative analysis of the RC circuit 
with local and non-local fractional derivatives," Revista Mexicana de Fisica, vol. 64, no. 6, pp. 647-654, 2018.

10.J. Gómez-Aguilar, "Fundamental solutions to electrical circuits of non-integer order via fractional derivatives with and without singular kernels," The European Physical Journal Plus volume, vol. 133, p. 197, 2018.

11.N. Sene, "Stability analysis of electrical RLC circuit described by the CaputoLiouville generalized fractional derivative," Alexandria Engineering Journal, vol. 59, pp. 2083-2090, 2020.

12.N. Sene, "Fractional input stability for electrical circuits described by the Riemann-Liouville and the Caputo fractional derivative," AIMS Mathemathics, vol. 4, no. 1, pp. 147-165, 2019.

13.J. Gómez-Aguilar, J. Rosales-Garcia, J. Razo-Hernandez and G.-C. M, "Fractional RC and LC electrical circuits," Ingenierias Investigación y Tecnología , vol. 15, no. 2, pp. 311-319, 2014.

14.M. Kenneth and B. Ross, An Introduction to the fractional Calculus and Fractional Differential Equations, Wiley-Blackwell, 1993. 